This item was submitted to Loughborough's Research Repository by the author.

Items in Figshare are protected by copyright, with all rights reserved, unless otherwise indicated.

Full-rate and full-diversity extended orthogonal space-time block coding in cooperative relay networks with imperfect synchronization

PLEASE CITE THE PUBLISHED VERSION

http://dx.doi.org/10.1109/ICASSP.2010.5496167

PUBLISHER

(c) IEEE

VERSION

VoR (Version of Record)

LICENCE

CC BY-NC-ND 4.0

REPOSITORY RECORD

Alotaibi, Faisal T., and Jonathon Chambers. 2019. "Full-rate and Full-diversity Extended Orthogonal Spacetime Block Coding in Cooperative Relay Networks with Imperfect Synchronization”. figshare.

https://hdl.handle.net/2134/7608. 
This item was submitted to Loughborough's Institutional Repository (https://dspace.lboro.ac.uk/) by the author and is made available under the following Creative Commons Licence conditions.

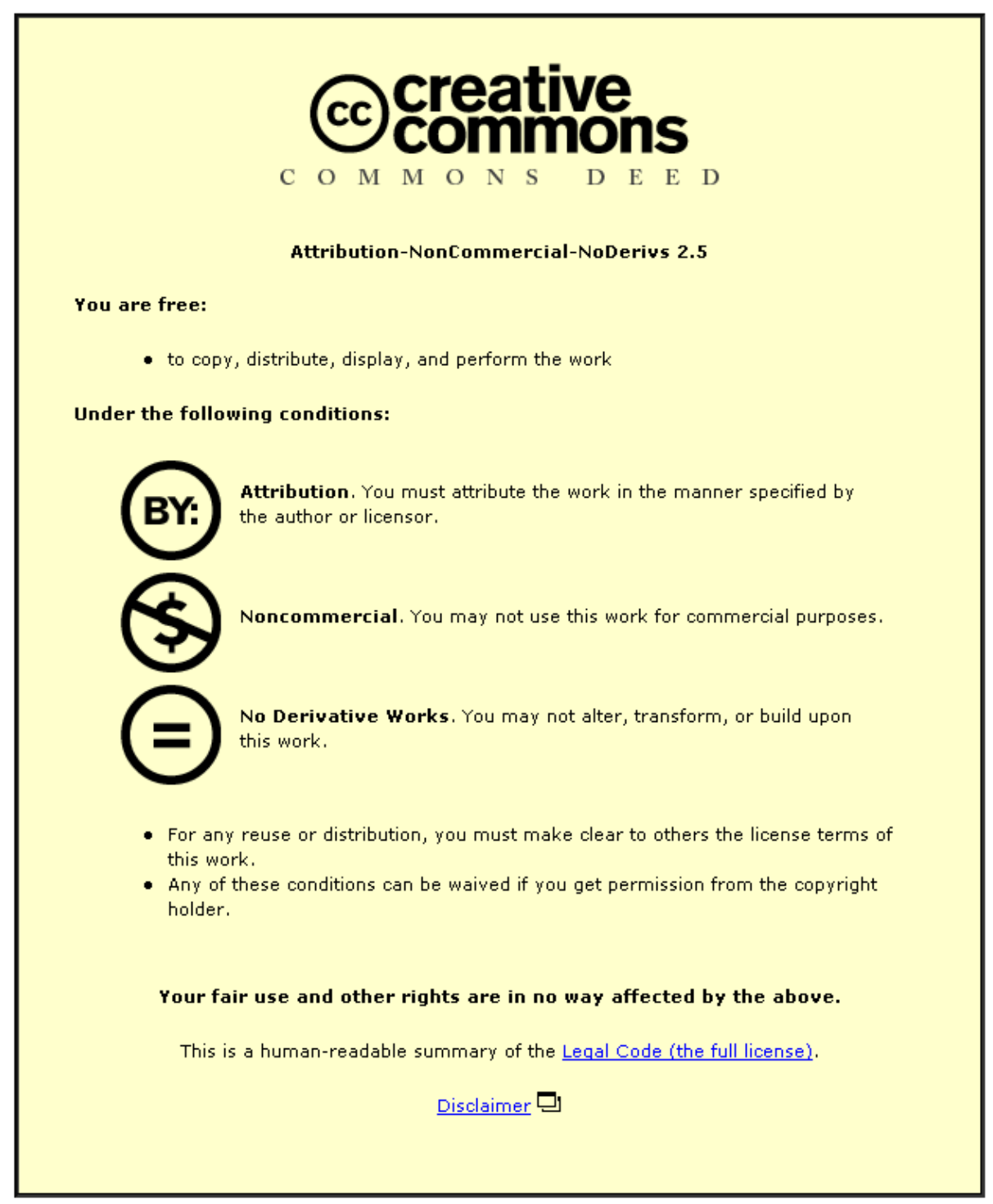

For the full text of this licence, please go to: http://creativecommons.org/licenses/by-nc-nd/2.5/ 


\title{
FULL-RATE AND FULL-DIVERSITY EXTENDED ORTHOGONAL SPACE-TIME BLOCK CODING IN COOPERATIVE RELAY NETWORKS WITH IMPERFECT SYNCHRONIZATION
}

\author{
F. T. Alotaibi and J. A. Chambers \\ Advanced Signal Processing Group, Department of Electronic and Electrical Engineering \\ Loughborough University, Loughborough LE11 3TU, UK \\ Email: $\{$ F.Alotaibi, J. A. Chambers $\} @ 1 b o r o . a c . u k$
}

\begin{abstract}
In this paper we present a novel extended orthogonal space-time block coding (EO-STBC) scheme for three and four relay nodes to use in asynchronous cooperative relay networks. This approach attains full-rate and full-diversity in that each hop attains unity rate and all four uncorrelated paths are utilized. Robustness against the effects of random delays at the relay nodes is enhanced through the use of a low-rate feedback channel. A new low complexity phase feedback scheme has been proposed which can retain the advantage of the perfect feedback scheme with substantial reduction in the feedback overhead. Orthogonal frequency division multiplexing (OFDM) with cyclic prefix (CP) is used at the source node to combat the timing errors at the relay nodes, which operate in a simple amplify-and-forward (AF) mode. Simulations show that our new scheme outperforms the previous schemes and uses a very simple symbol-wise maximum-likelihood (ML) decoder.
\end{abstract}

Index Terms - Asynchronized cooperative networks, cooperative diversity, EO-STBCs, distributed STBC, partial feedback.

\section{INTRODUCTION}

Next generation wireless communication systems will require efficient and cost effective approaches to meet increased performance demands. Cooperative communication is a promising approach that can effectively exploit the available communication network resources, improve range, increase data rate and mitigate the effect of fading without the need for multiple antennas at individual nodes [1]. It is desirable for systems where it may not be practical or even feasible due to equipment size and cost to employ multiple antenna array elements on the nodes. In particular, cooperative communications are based on exploiting the collection of distributed antennas belonging to multiple users to achieve cooperative diversity [1]. Recently, it has been shown that certain space-time block codes (STBCs) can be employed in a distributed fashion in single antenna relay networks to extract the same benefit as in point-to-point multi-input multi-output (MIMO) systems [1]. STBCs are attractive transmit diversity techniques that were pioneered by Alamouti [2] which can effectively exploit the diversity gains offered by point-to-point MIMO systems and certain cooperative MIMO systems with very low decoder complexity.

Because each relay node in a cooperative relay system is in a different location and has its own oscillator then synchronization between these nodes is hard to achieve. In this work the asynchronism considered is for the former case and is manifest in the variation of the frame delays from the different relays. Recently, there have been studies on space-time coding for such asynchronous cooperative re-

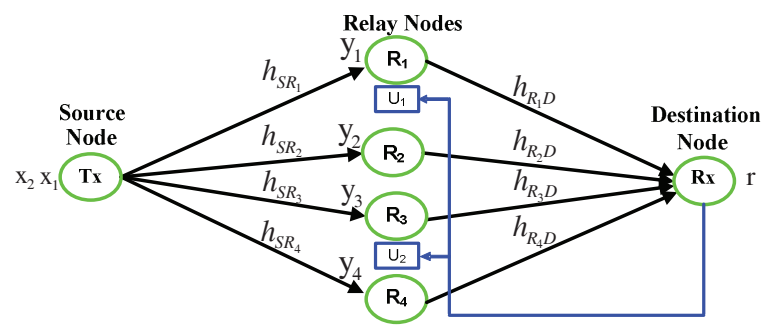

Fig. 1: Wireless relay network with single source and destination and four relay nodes together with feedback to two nodes.

lay systems; for example, [3], [4], [5], and [6]. Most of which use the decode-and-forward (DF) protocol.

In this paper, we propose increasing the robustness of a simple AF scheme for an asynchronous cooperative system proposed in [7] through the use of a low-rate feedback channel scheme. By applying feedback in the form of a phase rotation at the relay nodes as in [8] and [9], it is possible to extract full transmission rate and full cooperative diversity in addition to array gain from a complex orthogonal design. Also, a very simple ML decoding algorithm can be used at the decoder.

The remaining sections of this paper are organized as follows. Section 2 introduces the system model and description of the two-stage relaying protocol. The phase rotation techniques to handle the different delays along the paths through the four relay nodes are described in Section 3. In Section 4, the simulation results are presented. Finally, we draw conclusions in Section 5.

\section{EO-STBC SCHEME FOR ASYNCHRONOUS WIRELESS RELAY SYSTEMS}

Consider a wireless relay system with one source node, one destination node, and four half-duplex relay nodes, as shown in Fig. 1. Every node in the system has only one antenna. The system needs two stages to transmit the data from the source node to the destination node. In the first stage, the source node broadcasts the data to the four relay nodes $R_{i}, i=1,2,3,4$ and then stops sending during the second stage. In the second stage, the four relay nodes process the received signals and then retransmit them to the destination node. The channel between any two terminals is assumed to be quasi-static flat Rayleigh fading. The direct path between source and destination nodes is assumed to be unavailable due to path loss or shadowing effects. Denote the fading coefficients from the source node to the $i$ th relay as $h_{S R_{i}}$ and from the $i$ th relay to the destination node as $h_{R_{i} D}$. Assume that $h_{S R_{i}}$ and $h_{R_{i} D}$ are independent complex Gaussian random variables with zero-mean and unit-variance. 


\subsection{Implementation at the source node}

In the first stage the source node broadcasts sequentially two consecutive OFDM blocks $\mathbf{x}_{\mathbf{1}}=\left[x_{0,1}, x_{1,1}, \ldots, x_{N-1,1}\right]^{T}$ and $\mathbf{x}_{\mathbf{2}}=$ $\left[x_{0,2}, x_{1,2}, \ldots, x_{N-1,2}\right]^{T}$, which are composed of a set of $N$ modulated complex symbols $x_{i, j}$, which are converted into samples for transmission using IDFT and DFT operations, respectively, where $(.)^{T}$ represents the transpose operation and $j=1$ or 2 . So, $\mathbf{X}_{1}=$ $\operatorname{IDFT}\left(\mathbf{x}_{1}\right)$ while $\mathbf{X}_{2}=\operatorname{DFT}\left(\mathbf{x}_{2}\right)$. Then before broadcasting them to the four relays, each block is preceded by a CP with length $l_{c p}$. Thus, each OFDM symbol consists of $L_{s}=N+l_{c p}$ samples. Assume that $l_{c p}$ is not less than the maximum of the possible relative timing errors $\tau_{\max }$ of the signals arriving at the destination node. Denote two OFDM symbols $\mathbf{X}_{1}$ and $\mathbf{X}_{2}$ with the corresponding CP as $\overline{\mathbf{X}}_{1}$ and $\overline{\mathbf{X}}_{2}$.

\subsection{Implementation at the relay nodes}

The received signals at relay $i$ for two successive OFDM symbol durations can be written as

$$
\mathbf{Y}_{i j}=\overline{\mathbf{X}}_{j} h_{S R_{i}}+\mathbf{n}_{i j}
$$

where $\mathbf{n}_{i j}$ is the corresponding zero mean additive white Gaussian noise (AWGN) terms at relay node $i$ with elements having zeromean and unit-variance, in two successive OFDM symbol durations, respectively. Assume the channel coefficients are constant during two OFDM symbol intervals. Let $P_{s}$ denote the transmission power at the source node. Then the mean power of the signal $\mathbf{Y}_{i j}$ at a relay node is $P_{s}+1$ due to the unit variance assumption of the additive noise $\mathbf{n}_{i j}$ from the source node to a relay node in (1). Let $P_{r}$ denote the average transmission power at every relay node. The optimum power allocation proposed in [10] is used in this proposed scheme, which yields

$$
P_{s}=R P_{r}=\frac{P}{2}
$$

where $P$ is the total transmission power in the whole scheme and $R$ is the number of the relay nodes. The four relay nodes will process and transmit the received noisy signals according to the $i$ th column of the relay encoding matrix $\mathrm{S}$,

$$
S=\rho\left[\begin{array}{cccc}
\mathbf{Y}_{11} & \mathbf{Y}_{21} & -\mathbf{Y}_{32}^{*} & -\mathbf{Y}_{42}^{*} \\
\zeta\left(\mathbf{Y}_{12}\right) & \zeta\left(\mathbf{Y}_{22}\right) & \zeta\left(\mathbf{Y}_{31}^{*}\right) & \zeta\left(\mathbf{Y}_{41}^{*}\right)
\end{array}\right]
$$

where $\rho=\sqrt{\frac{P_{r}}{P_{s}+1}},(.)^{*}$ denotes the complex conjugation, and $\zeta($. represents the time-reversal of the signal, i.e., $\zeta(\mathbf{Y}(n))=\mathbf{Y}\left(L_{s}-\right.$ $n), n=0,1, \ldots, L_{s}-1$, and $\mathbf{Y}\left(L_{s}\right)=\mathbf{Y}(0)$.

According to the simple relay encoding scheme (3), the relay nodes only implement very simple operations on their received noisy signal in the form of: conjugation, amplification and reordering, and no decoding, DFT or IDFT operation is needed.

\subsection{Implementation at the destination node}

At the destination node, timing synchronization is easily implemented for the shortest path from source to destination. Without loss of generality, this will be assumed to be the path through relay 1. Hence, the delay in sample periods of the path through relay $i$ relative to that through relay 1 is denoted as $\tau_{i}$. To process the data, CP removal is performed for each OFDM symbol as in a conventional OFDM system as shown in Fig. 2. After the CP removal process, the reordering process needs to be performed on the second OFDM received frames to correct for the misalignment caused by the time

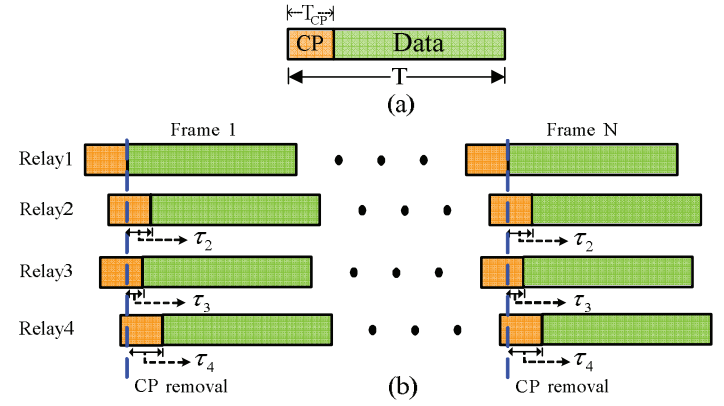

Fig. 2: (a) Frame structure. (b) CP removal with respect to relay 1 synchronization.

reversal in (3). This can be performed by shifting the last $l_{c p}$ samples of the $N$-point vector as the first $l_{c p}$ samples. After that, the received signals are transformed by the $\mathbf{N}$-point DFT. As mentioned before, because of the timing errors, the signals from relay nodes 2 , 3 , and 4 arrive at the destination node samples later than the signals from relay node 1 . Since $l_{c p}$ is not less than $\tau_{\max }$, the orthogonality between the subcarriers should still be maintained. The delay in the time domain corresponds to a phase change in the frequency domain

$$
\mathbf{f}^{\tau}=\left[f_{0}^{\tau_{i}}, f_{1}^{\tau_{i}}, \ldots, f_{N-1}^{\tau_{i}}\right]^{T}
$$

where $f_{k}^{\tau_{i}}=\exp \left(-j 2 \pi k \tau_{i} / N\right)$ and $k=0,1, \ldots, N-1$. Let the received signals for two successive OFDM blocks at the destination node after the CP removal and the DFT transformations be $\mathbf{Z}_{1}=$ $\left[Z_{0,1}, Z_{1,1}, \ldots, Z_{N-1,1}\right]^{T}$ and $\mathbf{Z}_{2}=\left[Z_{0,2}, Z_{1,2}, \ldots, Z_{N-1,2}\right]^{T}$. If we let $F_{1}=\operatorname{DFT}\left(\operatorname{IDFT}\left(\mathbf{x}_{1}\right)\right), F_{2}=\operatorname{DFT}\left(-\left(\operatorname{DFT}\left(\mathbf{x}_{2}\right)\right)^{*}\right), F_{3}=$ $\operatorname{DFT}\left(\zeta\left(\operatorname{DFT}\left(\mathbf{x}_{2}\right)\right)\right)$, and $F_{4}=\operatorname{DFT}\left(\zeta\left(\left(\operatorname{IDFT}\left(\mathbf{x}_{1}\right)\right)^{*}\right)\right)$, then $\mathbf{Z}_{1}$ and $\mathbf{Z}_{2}$ can be written as

$$
\begin{aligned}
\mathbf{Z}_{1}= & \rho\left[F_{1} h_{S R_{1}} h_{R_{1} D}+F_{1} \circ \mathbf{f}^{\tau_{2}} h_{S R_{2}} h_{R_{2} D}+F_{2}\right. \\
& \left.\circ \mathbf{f}^{\tau_{3}} h_{S R_{3}}^{*} h_{R_{3} D}+F_{2} \circ \mathbf{f}^{\tau_{4}} h_{S R_{4}}^{*} h_{R_{4} D}\right]+\mathbf{V}_{1} \\
\mathbf{Z}_{2}= & \rho\left[F_{3} h_{S R_{1}} h_{R_{1} D}+F_{3} \circ \mathbf{f}^{\tau_{2}} h_{S R_{2}} h_{R_{2} D}+F_{4}\right. \\
& \left.\circ \mathbf{f}^{\tau_{3}} h_{S R_{3}}^{*} h_{R_{3} D}+F_{4} \circ \mathbf{f}^{\tau_{4}} h_{S R_{4}}^{*} h_{R_{4} D}\right]+\mathbf{V}_{2}
\end{aligned}
$$

where $\circ$ is the Hadamard product and $\mathbf{V}_{j}=\rho \sum_{i=1}^{4} \mathbf{N}_{i j} \circ \mathbf{f}^{\tau_{i}} h_{R_{i} D}+$ $\mathbf{W}_{j}$ where $\mathbf{N}_{i j}=\left(N_{k, i j}\right)$ are the DFTs of $\mathbf{n}_{i j}$ and $\mathbf{W}_{j}=\left(W_{k, j}\right)$ are AWGN at the destination node with zero-mean and unit-variance. Using the identities $(\operatorname{DFT}(\mathrm{x}))^{*}=\operatorname{IDFT}\left(\mathrm{x}^{*}\right),(\operatorname{IDFT}(\mathrm{x}))^{*}=$ $\operatorname{DFT}\left(\mathrm{x}^{*}\right)$ and $\operatorname{DFT}(\zeta(\operatorname{DFT}(\mathrm{x})))=\operatorname{IDFT}(\operatorname{DFT}(\mathrm{x}))$, the $\mathbf{Z}_{1}$ and $\mathbf{Z}_{2}$ in (5) and (6) can be written as in the following EO-STBC form at each subcarrier $k$

$$
\left[\begin{array}{l}
Z_{k, 1} \\
Z_{k, 2}
\end{array}\right]=\rho \underbrace{\left[\begin{array}{cccc}
x_{k, 1} & x_{k, 1} & -x_{k, 2}^{*} & -x_{k, 2}^{*} \\
x_{k, 2} & x_{k, 2} & x_{k, 1}^{*} & x_{k, 1}^{*}
\end{array}\right]}_{X_{k}}\left[\begin{array}{l}
c_{k, 1} \\
c_{k, 2} \\
c_{k, 3} \\
c_{k, 4}
\end{array}\right]+\left[\begin{array}{l}
v_{k, 1} \\
v_{k, 2}
\end{array}\right]
$$

where $c_{k, i}=f_{k}^{\tau_{i}} h_{S R_{i}} h_{R_{i} D}$ and $v_{k, j}=\rho \sum_{i=1}^{4} N_{k, i j} f_{k}^{\tau_{i}} h_{R_{i} D}+$ $W_{k, j}$. Equivalently,

$$
\left[\begin{array}{c}
Z_{k, 1} \\
Z_{k, 2}^{*}
\end{array}\right]=\rho \underbrace{\left[\begin{array}{cc}
c_{k, 1}+c_{k, 2} & -\left(c_{k, 3}+c_{k, 4}\right) \\
\left(c_{k, 3}+c_{k, 4}\right)^{*} & \left(c_{k, 1}+c_{k, 2}\right)^{*}
\end{array}\right]}_{H_{k}}\left[\begin{array}{l}
x_{k, 1} \\
x_{k, 2}^{*}
\end{array}\right]+\left[\begin{array}{l}
v_{k, 1} \\
v_{k, 2}^{*}
\end{array}\right]
$$




\section{PHASE ROTATION SCHEMES FOR ASYNCHRONOUS WIRELESS RELAY SYSTEM}

From (8), it is clear that the equivalent channel matrix corresponding to the code word in (7) used over four relay nodes is given by:

$$
H_{k}=\left[\begin{array}{cc}
c_{k, 1}+c_{k, 2} & -\left(c_{k, 3}+c_{k, 4}\right) \\
\left(c_{k, 3}+c_{k, 4}\right)^{*} & \left(c_{k, 1}+c_{k, 2}\right)^{*}
\end{array}\right]
$$

Assuming matched filtering is performed at the destination receiver with the equivalent channel matrix in (9) for each of the k subcarriers, we can obtain the Grammian matrix $G_{k}$ as follows:

$$
G_{k}=H_{k}^{H} H_{k}=\left[\begin{array}{ll}
\alpha_{k} & 0 \\
0 & \alpha_{k}
\end{array}\right]
$$

where $(.)^{H}$ denotes Hermitian transpose and $\alpha_{k}$ represents the channel gain such that

$$
\alpha_{k}=\sum_{i=1}^{4}\left|h_{S R_{i}} h_{R_{i} D}\right|^{2}+\beta_{k, 1}+\beta_{k, 2}
$$

with $\beta_{k, 1}=2 \operatorname{Re}\left\{c_{k, 2}^{*} c_{k, 1}\right\}$ and $\beta_{k, 2}=2 \operatorname{Re}\left\{c_{k, 3} c_{k, 4}^{*}\right\}$ taking into account that $\left(f_{k}^{\tau_{i}}\right)^{*} f_{k}^{\tau_{i}}=1$ and $\beta_{k, 1}, \beta_{k, 2}$ are the interference factors, $|.|^{2}$ denotes the modulus squared of a complex number, and $\operatorname{Re}\{$.$\} its real part.$

From (10), it is evident that the extended STBC in (7) is orthogonal, which indicates that the code can be decoded with a simple receiver. It can be shown that the signal-to-noise ratio (SNR) is

$$
S N R=\alpha_{k} \frac{\sigma_{x}^{2}}{\sigma_{n}^{2}}=\left(\sum_{i=1}^{4}\left|h_{S R_{i}} h_{R_{i} D}\right|^{2}+\beta_{k, 1}+\beta_{k, 2}\right) \frac{\sigma_{x}^{2}}{\sigma_{n}^{2}}
$$

where $\sigma_{x}^{2}$ is the total transmit power of the desired signal, and $\sigma_{n}^{2}$ is the noise power at the receiver. As such, the $\beta_{k, 1}$ and $\beta_{k, 2}$ terms may reduce channel gain, and correspondingly the SNR. In order to leverage the system gain to the maximum, simple phase rotations, denoted by $U_{1}$ and $U_{2}$, are applied at two relays each having different code processing as proposed in [8]. The two relays that we selected in our system are $R_{1}$ and $R_{3}$ as shown in Fig. 1. We assumed that the perfect channel state information (CSI) is available at the destination receiver. So, before the signals are transmitted from the first and third relay nodes, they are multiplied by $U_{1}$ and $U_{2}$, respectively, while the other two are kept unchanged. Although the original implementation of [8] was designed for a single-carrier implementation, this can be extended to our scheme by applying the algorithm in [8] for each OFDM sub-carrier independently using the equivalent source to destination channel coefficients. The equivalent channel matrix $E_{k}$ is therefore given by

$$
E_{k}=\left[\begin{array}{cc}
U_{k, 1} c_{k, 1}+c_{k, 2} & -\left(U_{k, 2} c_{k, 3}+c_{k, 4}\right) \\
\left(U_{k, 2} c_{k, 3}+c_{k, 4}\right)^{*} & \left(U_{k, 1} c_{k, 1}+c_{k, 2}\right)^{*}
\end{array}\right]
$$

and consequently, $\alpha_{k}=\sum_{i=1}^{4}\left|h_{S R_{i}} h_{R_{i} D}\right|^{2}+\beta_{k, 1}+\beta_{k, 2}$ where $\beta_{k, 1}=2 \operatorname{Re}\left\{U_{k, 1} c_{k, 2}^{*} c_{k, 1}\right\}$ and $\beta_{k, 2}=2 \operatorname{Re}\left\{U_{k, 2} c_{k, 3} c_{k, 4}^{*}\right\}$ taking into account that $\left|U_{k, 1}\right|^{2}=\left|U_{k, 2}\right|^{2}=1$. The values of $\beta_{k, 1}$ and $\beta_{k, 2}$ after applying the feedback will be positive which means that the designed closed-loop system can obtain additional performance gain, which leads to an improved whole channel gain, and correspondingly the $S N R$ at the destination receiver.

The weighted values $U_{k, 1}$ and $U_{k, 2}$ are computed as in the following $U_{k, 1}=e^{j \theta_{k, 1}}$ and $U_{k, 2}=e^{j \theta_{k, 2}}$ [8]. So, we can propose the following design criteria:

$$
\theta_{k, 1}=-\operatorname{angle}\left(c_{k, 2}^{*} c_{k, 1}\right) \text { and } \theta_{k, 2}=-\operatorname{angle}\left(c_{k, 3} c_{k, 4}^{*}\right)
$$

In this case, the values of the two phase rotations are computed perfectly which requires many feedback bits which in practice may not be possible due to the very limited feedback bandwidth. Therefore, the phase angles should be quantized to minimize the overhead of the feedback channel. In this paper, we used two feedback strategies as in [8] and [9]. The first strategy based on quantizing $\theta_{k, 1}$ and $\theta_{k, 2}$ for each sub-carrier with assigns n-bits as a feedback for each subcarrier phase. So, the overhead of the channel is proportional to the OFDM symbol length and the number of bits that are used for the quantization process. In our simulation, we have used 2-bits feedback per sub-carrier phase quantization (2-bit FB/SCPQ) with the overhead of 128 bits, and 1-bit FB/SCPQ with the overhead of 64 bits. In the second feedback strategy, we propose a new low-rate feedback scheme termed as sub-carrier quantized phase linear interpolation considering discontinuity (SCQPLI-CDC). In this scheme, we use the same procedure as in the first strategy to quantize the phase rotation $\theta_{0, j}$ and $\theta_{1, j}$ (6-bits or higher for each) of the first and second sub-carriers denoted as $\phi_{0, j}$ and $\phi_{1, j}$ and send them back to the certain relay node. Then the relay node based on the two quantized phases and exploiting the linear relationship in (4) and considering the phase discontinuity in this relationship calculates the remaining sub-carriers phase rotations $\left[\begin{array}{lll}\phi_{2, j} & \phi_{3, j} \ldots \phi_{N-1, j}\end{array}\right]$. So,

$$
\phi_{k, j}=\phi_{k-1, j}+D_{j}
$$

where

$$
D_{j}= \begin{cases}\phi_{1, j}-\phi_{0, j} & \text { if }\left|\phi_{1, \mathrm{j}}-\phi_{0, \mathrm{j}}\right|>\left|\phi_{1, \mathrm{j}}-\phi_{0, \mathrm{j}}-2 \pi\right| \\ \phi_{1, j}-\phi_{0, j}-2 \pi & \text { otherwise }\end{cases}
$$

and if $\phi_{k, j}>\pi$ or $\phi_{k, j}<-\pi$, then

$$
\phi_{k, j}= \begin{cases}\phi_{k, j}+2 \pi & \text { if } \mathrm{D}_{\mathrm{j}}<0 \\ \phi_{k, j}-2 \pi & \text { otherwise }\end{cases}
$$

In our simulation, we have used 6-bits to quantize each of the first and second sub-carriers phase which results in 12 bits as the total overhead of the feedback channel.

Similarly, we can design a closed-loop scheme for three relay nodes with only one feedback link.

\section{SIMULATION RESULTS}

In Fig. 3, we show BER performance of the proposed closed-loop EO-STBC for four relay nodes. The BER performance of the proposed scheme with perfect feedback was compared with the previous AF Alamouti-OFDM scheme [7] and DF Alamouti-OFDM scheme [6]. In the simulation, the CSI is assumed to be perfectly known at the destination node and the length of the $\mathrm{CP}$ is 16 . The delay is chosen randomly from 0 to 15 with uniform distribution. The modulation used for the data symbols is BPSK to simplify the ML decoding process but it can be easily extended to complex constellations. The transmit power is fixed and identical for all schemes used in the comparison and the power allocation follows equation (2).

The results depicted in Fig. 3 confirm that our robust AF scheme significantly improves the BER performance over the previous schemes. For example, at a BER of $10^{-4}$, the proposed scheme requires approximately $17 \mathrm{~dB}$ while the $\mathrm{AF}$ and DF Alamouti-OFDM schemes require $27.5 \mathrm{~dB}$ and $26 \mathrm{~dB}$, respectively. Also it is clear that if one of the feedback channels or all is lost or one of the relay nodes fails 


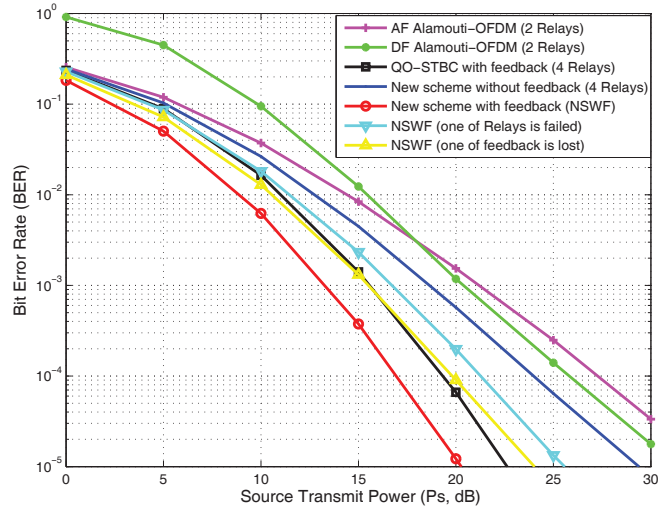

Fig. 3: Comparison of BER performance of the new AF asynchronous scheme using exact feedback channel information with previous asynchronous schemes in wireless relay system.

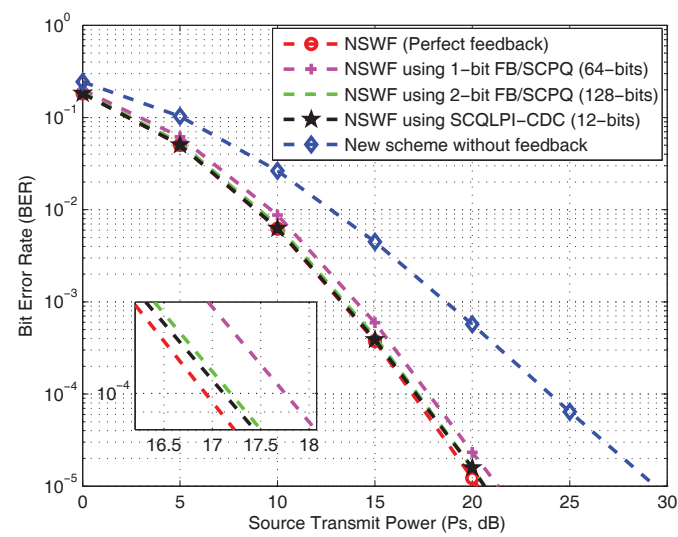

Fig. 4: Comparison of BER performance of the new AF asynchronous scheme using quantized channel information.

completely, our scheme improved BER performance over the AF and DF Alamouti-OFDM schemes for the whole source transmit power range; therefore ensuring a more robust transmission scheme.

The figure also provides a comparison of the proposed scheme with closed-loop quasi-orthogonal STBC (QO-STBC) [9] for four relays, where we clearly notice that the proposed scheme outperforms closedloop QO-STBC. In particular, at a BER of $10^{-4}$, our proposed scheme provides approximately $2.5 \mathrm{~dB}$ improvement. This improvement is because in our scheme we used the feedback to leverage the channel gain to the maximum so there is full cooperative diversity gain of order four and array gain while in QO-STBC scheme it was used to achieve the orthogonality of the transmission code so there is only full cooperative diversity. In addition to that, our scheme requires only half of the required time for QO-STBC scheme to decode the transmission data.

The performance of our scheme when we use 2-bit FB/SCPQ, 1-bit FB/SCPQ, and the new low-rate SCQLPI-CDC feedback schemes is depicted in Fig.4. Very little degradation in BER performance in the case of using low-rate SCQLPI-CDC scheme is shown compared with the performance of the scheme when we use un-quantized feedback while a slight decrease in the performance is observed in the case of using 2-bit FB/SCPQ and 1-bit FB/SCPQ schemes. Furthermore, we can see that the performance of our new scheme outper- forms the 64-bits and 128-bits feedback schemes. For example, at a BER of $10^{-4}$, our low-rate feedback scheme requires approximately $17.1 \mathrm{~dB}$ while the 64-bits and 128-bits feedback schemes require $17.8 \mathrm{~dB}$ and $17.2 \mathrm{~dB}$, respectively, although our scheme only used 12-bits for feedback. So, the new low-rate scheme achieves both better BER performance and a substantial reduction in the feedback overhead, i.e. a saving of $90.6 \%$ as compared with 2-bit FB/SCPQ scheme. However, the schemes with quantized feedback still provide significantly better performance than that of the previous schemes.

\section{CONCLUSIONS}

In this paper, we proposed a novel orthogonal space-time transmission scheme for use in asynchronous cooperative relay networks utilizing a low-rate feedback channel to the relay nodes. Simulations showed that the new scheme provides better performance than the previous schemes. The new closed-loop scheme achieves full cooperative diversity and array gain which results in improving the robustness of the wireless link between the source and destination nodes in the presence of fading and timing offset. Also, in this paper we present a low-rate SCQLPI-CDC feedback scheme which can retain the advantage of perfect feedback scheme with very limited feedback overhead. These result are very encouraging since the transmit power is the same in addition to the information symbols can be decoded separately in a very simple manner at the receiver.

\section{REFERENCES}

[1] J. N. Laneman and G. W. Wornell, "Distributed space-time coded protocols for exploiting cooperative diversity in wireless networks," IEEE Trans. on Information Theory, vol. 49, pp. 2415-2425, Oct. 2003.

[2] S. M. Alamouti, "A simple transmit diversity technique for wireless communications," IEEE J.Select. Areas Commun., vol. 16, pp. 1451-1458, Oct. 1998.

[3] Y. Li and X. G. Xia, "A family of distributed space-time trellis codes with asynchronous cooperative diversity," IEEE Trans. Commun., vol. 55, pp. 790-800, April 2007.

[4] S. Wei, D. L. Goeckel, and M. C. Valenti, "Asynchronous cooperative diversity," IEEE Trans. Wireless Commun., vol. 5, pp. 1547-1557, June 2006.

[5] X. Li, "Space-time coded multi-transmission among distributed transmitters without perfect synchronization," IEEE Signal Process. Lett., vol. 11, pp. 948-951, Dec. 2004.

[6] Y. Mei, Y. Hua, A. Swami, and B. Daneshrad, "Combating synchronization errors in cooperative relays," in Proc. IEEE ICASSP 2005, vol. 3, pp. 369-372, Mar. 2005.

[7] Z. Li and X. G. Xia, "A simple Alamouti space-time transmission scheme for asynchronous cooperative systems," IEEE Signal Process. lett., vol. 14, pp. 804-807, Nov. 2007.

[8] F. T. Alotaibi and J. A. Chambers, "Extended orthogonal space time block codes in wireless relay networks," IEEE Workshop on Statistical Signal Processing, Cardiff, UK, 2009.

[9] M. Hayes, J .A. Chambers, and M. D. Macleod, "A simple quasi-orthogonal space-time scheme for use in asynchronous virtual antenna array enabled cooperative networks," $E U$ SIPCO 2008, Lausanne, Swizerland, August 2008.

[10] Y. Jing and B. Hassibi, "Distributed space-time coding in wireless relay networks," IEEE Trans. Wireless Commun., vol. 5, pp. 3524-3536, Dec. 2006. 